\title{
Crisis e intercooperación: \\ las cooperativas de segundo o ulterior grado como instrumento de colaboración empresarial
}

\author{
Enrique Gadea \\ Universidad de Deusto
}

Recibido: 20.05.10

Aceptado: 07.06 .10

Sumario: 1. Concepto y características. 2. Capacidad, ingreso y baja de socios. 3. Régimen económico. 4. Estructura orgánica y derecho de voto. 5. Liquidación. 6. Normativa supletoria.

Resumen: La regulación actual de las CSG en nuestras Leyes más modernas se caracteriza por la amplitud funcional. Es por ello por lo que la colaboración empresarial podrá ser tan intensa como admitan las entidades que las integran, con el límite de no anular o prescindir de la fisonomía de cada miembro agrupado. En cada caso, serán los estatutos los que establezcan el grado de vinculación entre las entidades participantes. rial.

Palabras clave: Cooperativas de segundo grado y colaboración empresa-

Abstract: The current regulation of Second Degree Cooperatives in our most recent laws is characterised by the breadth of its functional scope. This is why business partnerships can be as intense as the entities that comprise them allow them to be, as long as they do not destroy or disregard the features of each of their group members. The articles of association will determine the extent of the links between the participating entities in each case.

Key words: Second degree cooperatives and business partnership. 


\section{Concepto y características}

Con la figura de las cooperativas de segundo (en los sucesivo CSG) o ulterior grado se pretende atender la necesidad de una efectiva colaboración empresarial. La CSG constituye un tipo legal de sociedad cooperativa en torno a la cual se organizan la mayor parte de los procesos de integración cooperativa ${ }^{1}$.

Pueden definirse como aquellas sociedades cooperativas que se componen de cooperativas de la misma o distinta clase y, en su caso, de socios de trabajo, empresarios individuales y otras entidades, con el objeto de promover, coordinar y desarrollar fines económicos comunes de los socios, y reforzar e integrar la actividad económica de los mismos (art. 77.1 LCOOP).

La regulación actual de las CSG en nuestras Leyes más modernas se caracteriza por la amplitud funcional2, por lo que la colaboración empresarial podrá ser tan intensa como admitan las entidades que las integran, aunque sin llegar a anular o prescindir de la fisonomía de cada miembro agrupado. En cada caso, serán los estatutos los que establezcan el grado de vinculación entre las entidades participantes, pudiendo ésta limitarse a una mera colaboración o a alcanzar una concentración empresarial en sentido propio.

Como ha señalado EMBID IRUJO3 al abordar el estudio de esta materia en la LCPV, queda reservada a la autonomía de la voluntad, consignada en los estatutos, la determinación concreta del modo de vinculación querida, así como su grado de intensidad, en el marco de la amplia configuración del fin social establecido por el legislador. Así, el art. 128.1. ${ }^{\circ}$ de la LCPV indica que «la cooperativa de segundo o ulterior grado tiene por objeto completar, promover, coordinar, reforzar o integrar la actividad económica de las entidades miembros y del grupo resultante en el sentido y con la extensión y alcance que establezcan los estatutos». Esta especie de indeterminación funcional de la cooperativa de segundo grado en la LCPV viene a confirmarse expresamente al advertir el legislador ciertas precisiones que deben constar en sus estatutos «cuando la cooperativa se constituya con fines de integración empresarial». Así, se mencionarán «las áreas de actividad empresarial integradas, la base para el ejercicio de la dirección unitaria del grupo y las características de éste (art. 128.1. in fine). La alusión final al grupo en términos inequívocos, así como

\footnotetext{
Parra de Mas, 1974, 143, Martínez Charterina, 1990, 42, y Embid Irujo, 1998, I, 8.

2 PANIAGUA, 2005, 322.

3 1998, II, 227.
} 
otras menciones en el precepto de esta modalidad de concentración empresarial, hace pensar en el deseo legislativo de convertir la CSG en "vestidura jurídica» de un grupo de sociedades (no necesariamente cooperativas, art. 129.1), aunque se trata de un criterio no exclusivo, sino meramente preferente.

\section{Capacidad, ingreso y baja de socios}

La CSG exige para su constitución, y para su funcionamiento, un mínimo de dos socios, que han de ser dos sociedades cooperativas (por todos, art. 77.1 LCCOP). Ahora bien, respecto a la capacidad para ser socio de una CSG o ulterior grado, hay que destacar la superación del diseño endogámico, según el cual sólo las cooperativas entre sí pueden constituir una entidad de este carácter de grado o nivel superior. En las Leyes vigentes más modernas (arts. 77.1 LCOOP, 129.1 LCPV, 122 LCCAT, 101 LCCV, 124.1 LCCM, 130.1 LCR, 158.1 LCEX y 130.1 LCG) podrán ser miembros de pleno derecho de estas sociedades, además de las cooperativas de grado inferior y los socios de trabajo, cualesquiera entidades de naturaleza pública o privada. Además, la LCOOP y la LCCM (arts. 77.1 y 124.1, respectivamente) admiten expresamente la presencia, como miembros de pleno derecho, de los empresarios individuales. También podrán incorporar, como las demás cooperativas, socios colaboradores a los que les será aplicable la normativa general (arts. 129.1 LCPV, 122 LCCAT y 124.1 LCCM).

Sin embargo, esta apertura no es incondicional, sino que está sujeta a determinadas limitaciones:

1. ${ }^{a}$ Se establecen medidas para garantizar que el control de la entidad de grado superior corresponda a las propias cooperativas. En ese sentido, en las diferentes Leyes se fija una limitación sobre el conjunto de votos que pueden poseer las entidades no cooperativas.

Así, en la LCPV (art. 129.1), en la LCCAT (art. 122) y en la LCEX (158.1) se señala que el conjunto de las personas jurídicas de naturaleza no cooperativa no podrá poseer más de la mitad del total de los votos de la cooperativa de segundo grado, sin perjuicio de que los estatutos puedan establecer un límite inferior.

En la LCCV (art. 101) se dispone que las personas jurídicas que no posean la forma cooperativa no podrán tener en la asamblea general un porcentaje superior al $40 \%$ de los votos presentes y representados.

En la LCCM (art. 124.1) el límite no podrá superar el 30\% del total de votos y en la LCG (130.6), el 25\%. 
En la LCOOP (art. 26.6) el número de votos de las entidades que no sean sociedades cooperativas no podrá alcanzar el $40 \%$ de los votos totales. Los Estatutos podrán establecer un límite inferior. Además, también se establece un límite sobre el porcentaje máximo del total de socios que pueden ser entidades no cooperativas. Sobre esta cuestión, se prevé que las personas jurídicas, públicas o privadas, y los empresarios individuales no pueden superar el $45 \%$ del total de socios (art. 77.1).

De ese modo, nuestra legislación incorpora la idea, manifestada en el Congreso de Manchester de 1995, de que basta con que las cooperativas al formar grupos tengan la mayoría. Sobre esta cuestión, el cuarto principio de "Autonomía e Independencia» señala, en efecto, que: "Las cooperativas son organizaciones autónomas de autoayuda gestionadas por sus socios. Si firman acuerdos con otras organizaciones, incluidos los gobiernos, o si consiguen capital de fuentes externas, lo hacen en términos que aseguren el control democrático por parte de sus socios y mantengan su autonomía cooperativa ${ }^{4}$.

2. ${ }^{a}$ La segunda limitación puede tener origen estatutario, dado que la posible pertenencia de las entidades no cooperativas y, en su caso, de los empresarios individuales puede ser prohibida por los estatutos. Esta posible limitación se recoge de manera expresa en determinadas Leyes Autonómicas (LCPV, LCCM, LCEX y LCG), aunque consideramos que es generalizable. Nótese que es obligatorio hacer constar en los estatutos de cualquier cooperativa «las clases de socios, los requisitos para su admisión y baja voluntaria u obligatoria y el régimen aplicable» (art. 11.1 j) LCCOP) ${ }^{5}$.

3. ${ }^{a}$ La tercera limitación deriva de la propia naturaleza de las cosas y alude a la necesaria convergencia de intereses y necesidades de índole económica de las personas jurídicas socios de naturaleza no cooperativa y, en su caso, de los socios empresarios individuales, con los demás socios de estructura cooperativa (se refieren a esta convergencia de forma expresa los arts. 129.1 LCPV, 124.1 LCCM, 158.1 LCEX, 18.1 LCG y 20.1 LCR). Decimos que esta limitación deriva de la propia naturaleza de las cosas porque el objeto de las CSG no es otro que promover, coordinar y desarrollar fines económicos comunes de sus socios, y reforzar e integrar la actividad económica de los mismos (así, art. 77.1 LCCOP).

Al igual que en las cooperativas de primer grado, la solicitud de admisión se formulará por escrito al consejo rector, que resolverá en un

\footnotetext{
4 Sobre el tema, García-Gutiérrez Revesco, 1995, 65-66.

5 Vázquez Pena, 2002, 71-72.
} 
plazo no superior al fijado por cada Ley (3 meses es el plazo fijado por el art. 13.1 de la LCCOP) a contar desde la recepción de aquélla, debiendo ser motivada la decisión desfavorable a la admisión. Sin embargo, en algunas Leyes de cooperativas que admiten la participación de entidades no cooperativas en las de segundo grado, se prevé que la admisión como socio de éstas requerirá acuerdo favorable del consejo rector por mayoría de al menos dos tercios de los votos presentes y representados, salvo previsión de otra mayoría - mayor o menor- en los estatutos (arts. 129.2 LCPV, 124.3 LCCM, 158.2 LCEX y 90 LCAR). Estas normas contemplan, además, la posibilidad de que los estatutos prevean para estos socios periodos de vinculación provisional o a prueba de hasta dos años; medida que permite comprobar su adaptación a las peculiaridades del grupo y a las particularidades de la estructuras cooperativa6.

La baja pone en peligro la subsistencia de la CSG, si se refiere a una de las dos cooperativas cuyo concurso es necesario para la constitución y el funcionamiento de la sociedad. En ese caso, la entidad podrá verse avocada a la disolución si en el plazo legal (6 meses en la LCCAT, LFCN o LCG y 1 año en la LCOOP, LCPV, LCA, LCCV o LCM) no incorpora otra sociedad de estructura cooperativa (o, como mal menor, a la transformación en una cooperativa de primer grado que absorba a las cooperativas socios en los términos del artículo 77.5 LCOOP). No obstante, sin necesidad de llegar a un caso tan extremo, es indudable que la baja de un socio puede acarrear daños al conjunto ${ }^{7}$. Por eso, para paliar los daños a los demás socios y a la propia entidad, determinadas Leyes (arts. 129.3 LCPV y 124.3 LCCM) establecen determinadas cautelas, que podían incorporar todas las normas, ante la baja de los socios personas jurídicas:

En primer lugar, el socio habrá de cursar un preaviso de al menos un año. Con ello, se amplia el período de preaviso normal relativo a las personas jurídicas de un año como máximo, a un año como mínimo.

En segundo lugar, antes de su efectiva separación estará obligado a cumplir las obligaciones contraídas con la cooperativa de segundo o ulterior grado o a resarcirla económicamente, si así lo decide el consejo rector de ésta.

En tercer lugar, salvo previsión estatutaria en contra, la entidad separada deberá continuar desarrollando, durante un plazo no inferior a dos años, aquellos compromisos adquiridos que hubieran asumido con anterioridad a la fecha de la baja.

6 Alfonso Sánchez, 2000, 475.

7 Embid Irujo, 1998, II, 229. 
Aspecto discutido, que merecería previsión legal, es la cantidad a reintegrar al socio que causa baja. Una postura mayoritaria considera que el socio de una cooperativa de segundo grado tiene derecho a participar en la cuota de liquidación, dado que, a excepción del FEP, todo el patrimonio de la cooperativa es repartible entre los socios ${ }^{8}$. Por el contrario, VICENT CHULIÁ ${ }^{9}$ se decanta por reconocer el clásico derecho al reembolso de las aportaciones, en las condiciones previstas en la ley y en los estatutos.

\section{Régimen económico}

En estas cooperativas es fundamental que los estatutos fijen con claridad los criterios o módulos que definen la actividad cooperativa, ya que es habitual (en la LCCM necesario ex art. 125.1) que las aportaciones obligatorias al capital social (y, por tanto, su porcentaje de participación en éste) se determinen en función de la actividad cooperativa comprometida con la entidad por cada socio; sin que su cuantía pueda superar, en su caso, el porcentaje máximo dispuesto en la ley.

Decimos «en su caso», porque el art. 77.1 de la LCOOP dispone que ningún socio de una CSG puede poseer más del 30\% del capital social de la entidad, salvo que nos encontremos ante una sociedad conjunta de estructura paritaria; mientras que la LCPV no impone limite alguno.

Junto a esta opción, la LCPV (art. 130.1) contempla la posibilidad de que las aportaciones obligatorias al capital social de una CSG se determinen en función del número de socios o conforme a los criterios establecidos para las cooperativas de primer grado en el art. 58.1 de la Ley.

También la distribución de resultados, tanto si son positivos como si se registran pérdidas, se acordará en función de la actividad cooperativa comprometida estatutariamente, después de haber realizado la imputación que proceda a los fondos de reserva y al fondo de educación y promoción (a éste último sólo si los resultados son positivos; arts. 130.2 LCPV y 125.2LCCM).

8 Paz Canalejo, 1990, 153; Trujillo Díez, 1999, 120-121; Alfonso Sánchez, 2000, 483.

9 1994, 294. 


\section{Estructura orgánica y derecho de voto}

El funcionamiento de las cooperativas de segundo o ulterior grado se articula, fundamentalmente, en torno a la asamblea general y al consejo rector (coincidimos con PAZ CANALEJO ${ }^{10}$ en que en las CSG no cabe administrador único).

En la asamblea general de la CSG participarán todos sus socios, pero unos lo harán por sí y otros por medio de representante. Las personas físicas, sólo podrán atribuir la representación a otro socio también persona física, con la excepción que, para los socios de trabajo, establecen la LCPV, LCCM y la LCEX (arts. 131.1, 126.1 y 160.1, respectivamente), en orden a la participación del colectivo a través de un solo representante. Por el contrario, la representación resultará obligada en caso de personas jurídicas, si bien tratándose de CSG reguladas por la LCOOP debe tenerse en cuenta que no pueden representar a las entidades socias las personas físicas que representen a las personas jurídicas en el consejo rector, interventores, comité de recursos y liquidadores (art. 77.3). En este punto, las normas difieren en cuanto a admitir la presencia de un solo representante por entidad (art. 158.2 LCCV, art. 130.3 LCG o art. 75.2 LCN) o de varios (art. 130.1 LCPV, 160.1 LCEX o art. 126.1 LCCM), siendo en este caso el criterio para la pluralidad de representantes el de la atribución de voto ponderado ${ }^{11}$.

A nuestro juicio, la pluralidad de representantes permite reflejar mejor la diversidad de posiciones en la asamblea, siendo posible el voto en distinto sentido por uno y por otro ${ }^{12}$. Precisamente, con la idea de fortalecer el carácter democrático de la CSG, el Grupo Parlamentario de Senadores Nacionalistas Vascos incorporó a la redacción original del entonces Proyecto de Ley de Cooperativas la frase que en la actualidad constituye el inciso final del art. 77.3 de la LCOOP de 1999: «excepto cuando en su composición las Entidades socios estén representados por varios miembros» (Enmienda núm. 95: vid. BOCG, Senado, VI Legislatura, Serie II: Proyectos de Ley, núm. 136, de 17 de mayo de 1999).

Es cierto que algunas normas parten del principio de un socio un voto (así, art. 101.3 LCCV) y que otras fijan de forma imperativa el criterio del voto proporcional (así, arts. 131 LCPV, 125.1 LCCM y 159.1 LCEX), pero todas permiten que los estatutos prevean el voto proporcional a la participación de cada socio en la actividad cooperativizada de la CSG y/o al número de socios de cada entidad asociada.

\footnotetext{
$102002,185$.

11 Alfonso Sánchez, 2000, 494.

12 Vicent Chuliá, 1978, 457-458.
} 
En cuanto al límite cuantitativo al derecho de voto, coexisten en nuestras Leyes tres sistemas:

1. Aquél en el que las limitaciones al número de votos por socio se establece para todos los socios.

Ejemplo de esta orientación es la LCAND que en el art. 52.2 cifra el máximo por socio en el 50\% de los votos totales.

2. ${ }^{\circ}$ El que impone limitaciones al número de votos para todos los socios y, además, establece un límite global al número de votos de las entidades que no sean sociedades cooperativas.

En esta línea, el art. 26. 6 de la LCOOP señala que ningún socio podrá ostentar más de un tercio de los votos totales, salvo que la sociedad esté integrada sólo por tres socios, en cuyo caso el límite se elevará al cuarenta por ciento, y si la integrasen únicamente dos socios, los acuerdos deberán adoptarse por unanimidad de voto de los socios. En todo caso, el número de votos de las entidades que no sean sociedades cooperativas no podrá alcanzar el cuarenta por ciento de los votos totales. Los Estatutos podrán establecer un límite inferior.

3. El que impone límites de votos por socio únicamente a las entidades que no sean sociedades cooperativas.

Así, el art. 131.1 de la LCPV señala que el número de votos de una entidad que no sea cooperativa no podrá ser superior a un tercio de los votos sociales, aunque no regirá esta limitación cuando hubiese menos de cuatro socios.

Esta última orientación ha sido criticada por EMBID IRUJO13, por considerar que lleva implícito el riesgo de que una sola entidad cooperativa se alce con el control de una entidad de segundo grado. Ese riesgo se evita fijando un doble criterio de limitación como el establecido por la LCOOP.

Respecto a las particularidades del consejo rector, anotar, en primer lugar, que con las reformas de la legislación cooperativa se han ampliado sus posibles miembros. Así, de la exigencia de que los miembros debieran ser socios de las cooperativas de base, se ha pasado a permitir que lo sean los propios miembros de la cooperativa secundaria, e incluso, si lo prevén los estatutos, terceros no socios (arts. 77.2 LCOOP, 131.2 LCPV, 130.4 LCG, 126.2 LCCM y 101) ${ }^{14}$. La limitación a la incorporación de terceros no socios en el consejo rector de las CSG

13 1998, II, 230.

14 Sobre la incorporación de consejeros no socios, Tusquets Trías de Bes, 2000, 5657. 
se fija en que necesariamente sean mayoría los administradores socios en la LCCV, en un tercio de sus miembros totales en las LCOOP, LCPV y LCCM y en un $25 \%$ en la LCG.

Para garantizar la representación de todas las entidades socias, normalmente, se opta por un consejo formado por tantos consejeros como socios tenga la CSG, aunque para evitar consejos demasiado numerosos, determinadas normas (art. 131.1 LCPV y 126.2 LCCM) han cifrado en quince el número máximo de integrantes. En el caso de que el número de entidades socias superase las quince, permiten la agrupación de las entidades de menor número de votos para designar un representante.

En el seno del consejo es posible que rija la regla general y que cada consejero tenga un voto, aunque también cabe que el derecho de voto se fije en proporción a la actividad cooperativa o al número de socios de la entidad o entidades a las que representen los consejeros.

\section{Liquidación}

En caso de disolución con liquidación de una cooperativa de segundo o ulterior grado, la normativa cooperativa contiene especialidades al regular el destino del fondo de reserva y del haber líquido resultante. Sin embargo, la LCOOP —en el art. 77.4- se refiere, como destinatarias únicas de tales cantidades, a las cooperativas socios, olvidando a los socios de naturaleza no cooperativa.

En efecto, el art. 77. 4 LCOOP señala que «en el supuesto de liquidación, el fondo de reserva obligatorio se transferirá al fondo de la misma naturaleza de cada una de las cooperativas que la constituyen, así como el resto del haber líquido resultante, distribuyéndose todo ello entre las cooperativas socios en proporción al volumen de la actividad cooperativizada desarrollada por cada una de ellas en la cooperativa de segundo grado durante los últimos cinco años o, en su defecto, desde la constitución, no teniendo carácter de beneficios extracooperativos».

Esta exclusión constituye un grave error motivado por el arrastre indebido de la legislación anterior, en la que estaba casi absolutamente prohibida la presencia de empresas no cooperativas en una CSG. Pero una interpretación integradora (en base al art. 3.1 del CC) debe superar la literalidad de la norma; lo contrario supone dar pie a una «expropiación legislativa» de derechos económicos de los socios de naturaleza no cooperativa. No tiene ningún sentido convertir en extraños a quienes eran socios de pleno derecho de la CSG, una vez que se les hayan 
restituido sus aportaciones al capital social. Otro planteamiento es injusto e incoherente con la apertura de la CSG a sociedades o empresarios de otra naturaleza ${ }^{15}$.

En este punto, una solución adecuada debe pasar por la redacción de un precepto sin exclusiones, con un tenor literal semejante al siguiente: "En caso de disolución con liquidación de una cooperativa de segundo o ulterior grado, el activo sobrante será distribuido entre los socios en proporción al importe del retorno percibido en los últimos cinco años o, para las cooperativas cuya duración hubiese sido inferior a este plazo, desde su constitución. Si la cooperativa no hubiese repartido retornos, su activo sobrante se distribuirá, según determinen los estatutos o el reglamento de régimen interno, bien en proporción a la participación de cada socio en la actividad cooperativizada o bien al número de miembros activos de cada entidad agrupada en aquella cooperativa, pero sin excluir a los socios individuales, sean usuarios o de trabajo» (en sentido similar, arts. 132 LCPV y 127 LCCM).

\section{Normativa supletoria}

El artículo 77.6 de la LCOOP al trazar el cuadro de fuentes jurídicas aplicables a las CSG, señala que, en lo no previsto en el mismo, estas cooperativas «se regirán por la regulación de carácter general establecida en esta Ley en todo aquello que resulte de aplicación». Al principio, el precepto fue muy criticado por considerar que ordenaba una rígida e inmediata aplicación supletoria de la normativa sobre cooperativas de primer grado. Sin embargo, en la actualidad, no faltan autores que consideran que con el tenor literal del precepto no se limita la capacidad de autorregulación de las $\mathrm{CSG}^{16}$. Consideran que el recurso a la vía estatutaria es posible, dado que en el artículo 77.6 se reconoce que no toda la regulación de carácter general establecida en la Ley resulta de aplicación a las CSG. Por ello, cuando no resulte aplicable esa regulación, el régimen de la CSG se habrá de integrar con las previsiones estatutarias, que, en todo caso, deberán respetar la Ley y los principios configuradores de la forma societaria cooperativa.

Como ha señalado EMBID IRUJO ${ }^{17}$, la singularidad de estas entidades puede exigir algunos preceptos específicos que, tal vez, no pueden

15 Paz Canalejo, 2002, 189.

16 Alfonso Sánchez, 2000, 365-366, Vázquez Pena, 2002, 51, y Paz Canalejo, 2002, 177-178; en contra, Cuenca García, 2000, 73.

17 1991, 42. 
obtenerse con remisión a las normas generales de la ley. Por ello, deben ser los estatutos los encargados de llenar todas las lagunas existentes en el ordenamiento, con la salvedad de que todas las cláusulas deberán ser contrastadas con los principios generales de la ley.

Lo anterior, sin perjuicio de reconocer que la legislación estatal ha optado por una regulación menos avanzada que la del País Vasco (seguida, posteriormente, por la LCCM y LCEX, arts. 128 y 162, respectivamente), que se decanta, sin reservas, por la flexibilidad de cauce autorregulador para completar las previsiones legales.

El artículo 133 de la LCPV establece que «en lo no previsto por los artículos anteriores de esta sección, se estará a los establecido en los estatutos y en el reglamento de régimen interno y, en su defecto, en cuanto lo permita la especifica función y naturaleza de las cooperativas de segundo o ulterior grado, a lo establecido en la presente ley sobre cooperativas de primer grado»

En efecto, bajo ese régimen legal, la CSG puede optar entre varias fórmulas: desarrollar todo el marco legal en los estatutos; prever sólo unas pautas estatutarias de carácter mínimo (incluyendo únicamente aquellas materias que la Ley obliga a regular en los estatutos) y completar aquellas pautas con un reglamento de régimen interno muy analítico; o bien, finalmente, redactar un estatuto relativamente extenso, pero no exhaustivo, y completarlo con un reglamento interno de reducido alcance ${ }^{18}$.

\section{Biblíografía}

Alfonso SÁnchez, La integración cooperativa y sus técnicas de realización: las cooperativas de segundo grado, Tirant lo Blanch, Valencia, 2000.

Cuenca García, "Las cooperativas de segundo grado en la Ley 27/1999, de 16 de julio, de cooperativas, CIRIEC, núm. 11, 2000, pp. 69-118.

EMBID IRUjo, Concentración de empresas y Derecho de cooperativas, Universidad de Murcia, Murcia, 1991.

EMBiD IRUjo, «Problemas actuales de la integración cooperativa», RDM, núm. 227, 1998, I, pp. 7 y ss.

EMBID IRUjo, "La integración cooperativa y su tratamiento en la Ley 4/1993, de 24 de junio, de cooperativas de Euskadi», en AA.VV., Estudios de Derecho Mercantil. Homenaje al Profesor Justino F. Duque, vol. I, Universidad de Valladolid, Valladolid, 1998, II, pp. 223 y ss.

18 Paz Canalejo, 1999, 472-473. 
García-Gutiérrez Fernández, "Las sociedades cooperativas de derecho y las de hecho con arreglo a los valores y a los principios del Congreso de la Alianza Cooperativa Internacional de Manchester de 1995: especial referencia a las sociedades de responsabilidad limitada reguladas en España», REVESCO, 1995, núm. 61, pp. 53 y ss.

Martínez Charterina, Análisis de la integración cooperativa, Universidad de Deusto, Bilbao, 1990.

PARRA DE MAS, La integración de la empresa cooperativa (Evolución de los principios cooperativos), Editorial de Derecho Financiero, Madrid, 1974.

Paz Canalejo, "Comentario al Capítulo X», en Glosa a la Ley de cooperativas de Euskadi, VV.AA., Consejo Superior de Cooperativas de Euskadi, VitoriaGasteiz, 1999.

Paz Canalejo, La sociedad cooperativa ante el reto de los mercados actuales (un análisis no sólo jurídico), Ministerio de Trabajo y Asuntos sociales, Madrid, 2002.

Paz Canalejo Y Vicent Chulia, "Ley General de cooperativas», tomo XX, vols. 2 y 3, en Comentarios al Código de comercio y Legislación mercantil especial, dirigido por Sánchez Calero y Albaladejo, Edersa, Madrid, 1990, 1994.

TRUJILlo DíEZ, «Baja del socio cooperativo y reembolso de sus aportaciones sociales», AC, 1999, núm. 4, pp. 113 y ss.

TUSQUETS TRÍAS DE BES, «La profesionalización de los cargos directivos en la nueva Ley de cooperativas», RGD, 2000, núms. 664-665, pp. 49-60.

Vázquez Pena, Las cooperativas de segundo grado: peculiaridades societarias, Tirant lo Blanch, Valencia, 2002.

VICENT ChulíA, «La asamblea general de la cooperativa, RJC, 1978, núm. 2, pp. 417 y ss. 